13

\title{
Dosage of estradiol, bone and body composition in Turner syndrome: a 5-year randomized controlled clinical trial
}

\author{
Line Cleemann', Kirsten Holm', Hanne Kobbernagel2 ${ }^{2}$ Bent Kristensen², \\ Sven Oluf Skouby ${ }^{3}$, Andreas Kryger Jensen ${ }^{4,5}$ and Claus H Gravholt ${ }^{6,7}$ \\ 'Department of Pediatrics and 2Department of Radiology, Nordsjællands Hospital, Hillerød, Denmark, \\ ${ }^{3}$ Department of Gynecology and Obstetrics, Herlev University Hospital, Herlev, Denmark, ${ }^{4}$ Center of \\ Research and Innovation, Nordsjællands Hospital, Hillerød, Denmark, ${ }^{5}$ Department of Public Health, \\ University of Copenhagen, Copenhagen, Denmark, ${ }^{6}$ Department of Endocrinology and Internal \\ Medicine and the Medical Research Laboratories, Aarhus Sygehus NBG, Aarhus University Hospital, \\ Aarhus C, Denmark, and ${ }^{7}$ Department of Molecular Medicine, Aarhus University Hospital, \\ Aarhus N, Denmark
}

\author{
Correspondence \\ should be addressed \\ to L Cleemann \\ Email \\ line.cleemann@regionh.dk
}

\begin{abstract}
Objective: Reduced bone mineral density (BMD) is seen in Turner syndrome (TS) with an increased risk of fractures, and body composition is characterized by increased body fat and decreased lean body mass. To evaluate the effect of two different doses of oral 17B-estradiol in young TS women on bone mineral density (BMD), biochemical markers of bone turnover and body composition with the hypothesis of a positive effect of the higher dose.

Design: A double-blind 5-year randomized controlled clinical trial. 20 young TS women participated. Inclusion criteria were diagnosis of TS, age 15-25 years and current treatment with $2 \mathrm{mg}$ oral estradiol daily.

Methods: The low-dose (LD) group was administered $2 \mathrm{mg}$ 17B-estradiol/day orally and placebo, the high-dose (HD) group was administered $2+2 \mathrm{mg}$ 17B-estradiol/day orally. Main outcome measures were whole body and regional bone mineral density (BMD), lean body mass (LBM), fat mass (FM) measured yearly by DXA scan and resorptive and formative bone markers in serum.

Results: BMD, whole body and regional, increased over time with an attenuation toward the end of the study, and bone turnover markers decreased over time, both with no differences between the treatment groups $(P=0.2-0.9)$. LBM increased significantly more in the HD group $(P=0.02)$. FM remained stable in both groups.

Conclusions: A steady increase in BMD over time in TS was found similar to healthy young women. The higher estrogen dose did not differentially affect BMD or bone markers. The positive effect on body composition may have long-ranging health benefits in TS.
\end{abstract}

European Journal of

Endocrinology

(2017) 176, 233-242

\section{Introduction}

Ovarian failure occurs in the majority of girls with Turner syndrome (TS) and supplementation with estrogen during puberty and throughout adulthood remains the mainstay of treatment (1).

Unequivocal epidemiological clinical studies have shown that fracture risk in TS is increased $(2,3)$. Results of previous studies of bone mineral density (BMD) in girls
() 2017 European Society of Endocrinology Printed in Great Britain and adolescents with TS are conflicting, and it may in part be due to methodological differences and limitations (4). Substantial improvement with normalization or near normalization in BMD values are obtained when appropriate adjustments are made for the reduced bone size in TS $(1,5,6)$, and subsequent studies reporting adjusted BMD have not identified a significant problem

Published by Bioscientifica Ltd. 
in the pediatric age group (7). In adult TS women, studies using high-resolution pQCT have recently identified compromised trabecular microarchitecture and reduced bone strength, which may explain the increased fracture risk (8). At the same time, body composition in TS is altered in an unfavorable direction with lower muscle mass and greater fat mass in TS $(9,10)$, possibly in concert exerting a negative impact on bone strength $(11,12,13,14)$.

Hormonal replacement therapy (HRT) using estrogen doses that raise serum estradiol levels to the upper end of the normal range may exert an anabolic effect on bone and stimulate bone formation in addition to the antiresorptive effect, whereas lower levels primarily affect bone resorption (15). Estradiol may also exert a weak anabolic effect on muscle mass without affecting fat mass $(9,16)$.

Markers of bone metabolism may account for differences in the TS bone phenotype. An impaired coupling of bone formation and resorption in girls with TS seems to exist $(17,18)$.

In a former cross-sectional study, we found a normal tempo of bone formation in a large group of girls and adolescent with TS compared to healthy controls despite signs of accelerated bone resorption (19). In the present prospective study, we hypothesized that high-dose oral 17B-estradiol in young women with TS would have beneficial effects on BMD and body composition and correct the imbalance between bone formation and bone resorption.

\section{Subjects and methods}

\section{Design}

A 5-year prospective double-blind randomized controlled clinical trial using a parallel group design. TS patients fulfilling the inclusion criteria were randomized into one of two study groups, assigned to take estrogens as follows: the lower dose (LD) group took Trisekvens (day 1-12: $2 \mathrm{mg}$ estradiol; day 13-22: $2 \mathrm{mg}$ estradiol and $1 \mathrm{mg}$ norethisteronacetat; day 23-28: $1 \mathrm{mg}$ estradiol) (Novo Nordisk) orally combined with placebo on day 1-22 of the menstrual cycle. The higher dose (HD) group took Trisekvens (Novo Nordisk) orally combined with estradiol $2 \mathrm{mg}$ (Novo Nordisk) on day 1-22 of the menstrual cycle.

All participants went through examinations at baseline and thereafter yearly within a time limit of \pm 4 weeks for a total of 5 years. Body weight and height were measured, and body mass index (BMI) was calculated.
Body surface area (BSA) was calculated according to the formula of Dubois and Dubois $\left(\mathrm{BSA}\left(\mathrm{m}^{2}\right)=0.20247 \times\right.$ height $(\mathrm{m})^{0.725} \times$ weight $\left.(\mathrm{kg})^{0.425}\right)$. Pubertal development stage according to Tanner stages was noted.

The protocol was approved by the Institutional Review Board (the Research Ethics Committees of The Capital Region of Denmark) (H-Ø-2004-2-24G). The study was conducted according to the recommendations of the Declaration of Helsinki and the ICH guideline for Good Clinical Practice. The protocol was registered in the EudraCT database (2004-004778-99) and at ClinicalTrials.gov (NCT00134745). All participants (and her parents if age $<18$ years) received oral and written information concerning the study prior to giving written informed consent.

\section{Participants}

20 young women with TS (age: $19.2 \pm 2.5$ (mean \pm S.D.), range 16.0-24.9 years) and an age-matched control group participated, based on a power analysis (Supplementary data 1 , see section on supplementary data given at the end of this article).

The criterion for inclusion were diagnosis of TS confirmed by previous karyotyping $(45, X(n=8), 45 X / 46 X X$ $(n=2), 45 X / 46 X Y(n=3)$, iso-chromosomes $(n=3)$, and deletions $(n=4)$ ), age $15-25$ years, achieved final height and discontinued growth hormone $(\mathrm{GH})$ treatment and current treatment with an oral estradiol dose of $2 \mathrm{mg}$ daily. Exclusion criteria were untreated hypothyroidism or hyperthyroidism, severe or chronic disease or treatment with drugs known to interfere with bone metabolism, contraindications to the study medication and current use of other medication with known or potential interactions with the study medication. Individual case notes were reviewed and data were collected on the complete medical history with emphasis on puberty and menarche (spontaneous or induced), age at initiation of hormonal replacement therapy (HRT), types of estrogen, initial dose and subsequent increment doses used. Also a record of former treatment with growth hormone (GH) and androgen (Oxandrolone) was noted. Two participants decided to leave the study in the first year of treatment; one due to lack of compliance to the protocol and one due to side effects (edema of the lower limbs). One participant left the study in the second year of treatment due to a wish of pursuing pregnancy. One participant left the study in the fifth year of treatment for unknown reasons. No fractures were recorded during the study period. 
A control group of 34 healthy age-matched girls and young women (age: 18.4 \pm 2.7 , range 15.1-25.0 years) were recruited from local schools by advertisement and examined once. All had normal pubertal development.

\section{Blood sampling}

Blood was drawn from the TS participants evenly distributed throughout the calendar year, whereas the controls were examined in the summer. TS patients were examined in the morning after an overnight fast (12h), whereas controls were examined in the afternoon for logistical reasons. Urine was morning spot urine sample (second void) in all participants.

\section{Dual-energy X-ray absorptiometry (DXA)}

Using a Hologic QDR2000/w osteodensitometer (Hologic, Inc., Waltham, MA, USA), we measured total and regional FM ( $\mathrm{g}$ ) and LBM (g) in pencil-beam mode. The system software provided the mass of lean body, fat and bone mineral for the whole body. Bone mineral content (BMC) (grams) and bone mineral density (BMD) (grams/ square centimeter) were assessed as total body value and regional at the level of the lumbar spine $\left(\mathrm{BMD}_{\text {spine }}\right)$, the hip $\left(\mathrm{BMD}_{\text {hip }}\right)$ and the non-dominant forearm $\left(\mathrm{BMD}_{\mathrm{arm}}\right)$. The measurements at the arm were specified as radius ultra-distal (UD), radius middle (MiD) and proximal part of the distal third of radius (1/3). BMD values were reported in absolute values. Volumetric BMD at the spine $\left(\mathrm{vBMD}_{\text {spine }}\right)$ and hip $\left(\mathrm{vBMD}_{\text {hip }}\right)$ were calculated as previously described (18).

\section{Assays}

Plasma carboxy-terminal collagen crosslinks (CTX), plasma bone-specific alkaline phosphatase (BALP) and plasma Pro-collagen I amino-terminal pro-peptide (PINP) were measured.

Androstendione, 17B-estradiol, estrone, estrone sulfate, dihydrotestosterone (DHT) and 17OH-progesterone were measured. Levels of LH, FSH and SHBG were quantified separately (further details on assays in Supplementary data 1$)$.

\section{Statistical analysis}

Data were analyzed using linear mixed-effects regression for all outcome variables to account for the betweensubject dependencies. An initial non-parametric
(LOWESS) smoothing of data stratified by treatment suggested a non-linear development over time for the majority of the outcomes, and we therefore included 'time since randomization' in years as a continuous covariate in both linear and quadratic form and their interactions with treatment allocation as fixed effects. A main effect of treatment was omitted due to the randomized design. Correlated subject-specific intercepts and slopes were included as random effects.

For all outcome variables except FM, LBM and body fat percentage, we investigated the effect of selected background variables assumed to have the possibility of modifying the time development of the outcomes both within and between treatment groups. We included these as main effects and all interactions with linear time and treatment. These models were simplified with a standard backward procedure, and we only report a model if the background variable entered significantly in the reduction. For each outcome, the models were compared to each other with the Akaike Information Criterion (AIC).

The null-hypothesis of no effect of treatment was tested with a likelihood ratio test, and $P$ values below 5\% were considered significant. 95\% confidence intervals for the regression parameters were calculated using a parametric bootstrap approach with 5000 replicates and the percentile method. All data was analyzed as intention to treat, and missing observations were assumed to be missing at random (MAR). This approach allowed us to use observations from all individuals until the end of the study or until dropout, which occurred in some cases. The appropriateness of the models was assessed by standard residual diagnostic methods. For the analyses, we used R, version 3.2.2 and the lme4 package, version 1.1-9.

\section{Results}

\section{Study subject}

10 patients were allocated to the LD group and 10 patients to the HD group at baseline. The karyotype $45, X$ was equally distributed among the two groups $(n=4,40 \%)$. There were no significant differences between the two groups on relevant parameters at baseline (Supplementary Table 1). All patients except for one patient in the HD group had been treated with GH previously. Four patients in each group had spontaneous puberty and two patients in the LD group and one patient in the HD group had spontaneous menarche, but all subsequently suffered from premature ovarian failure. Tanner breast stage was similar (data not shown), and there was no difference in 
A

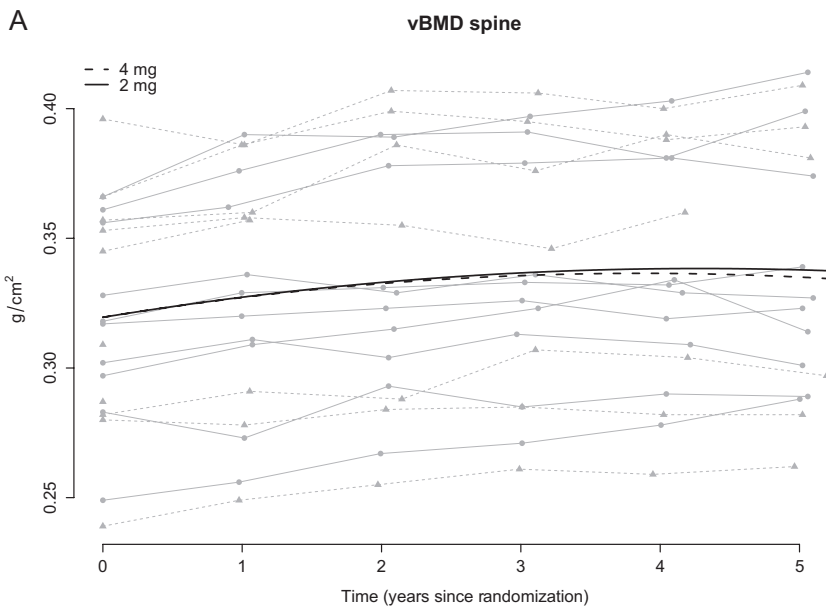

B
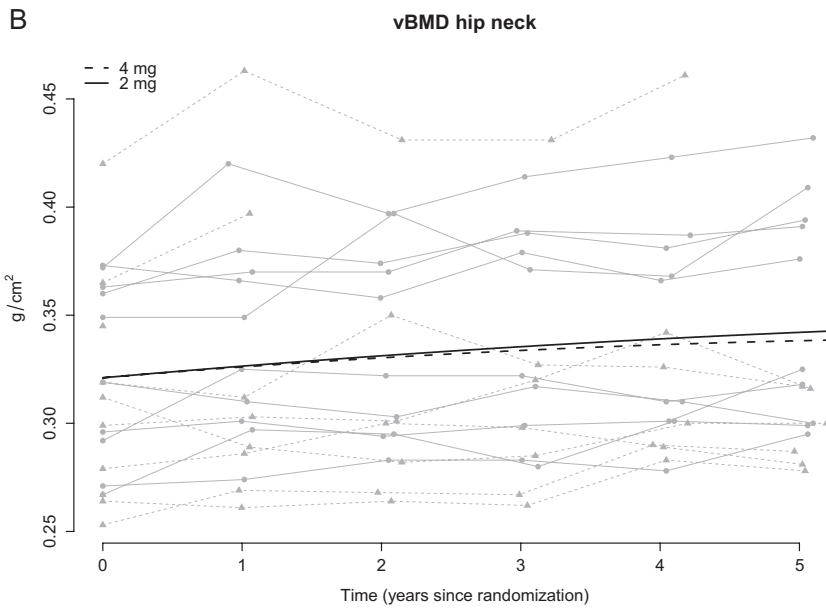

C

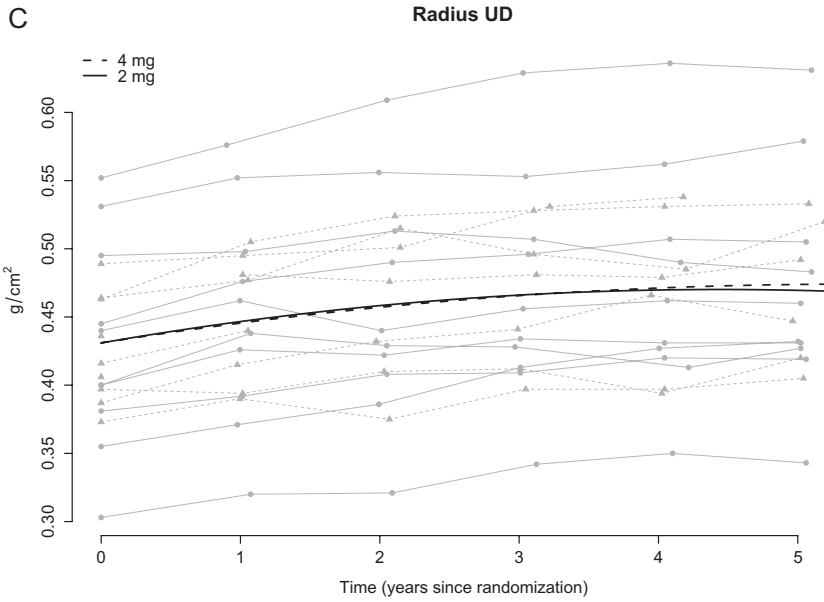

\section{Figure 1}

(A) Developmental changes in volumetric $\mathrm{BMD}_{\text {spine }}$ during the study period. Thin lines are the observed data for each patient, straight line (LD group), dotted line (HD group). Thick lines are estimated population means from the mixed model based on all data. (B) Developmental changes volumetric $B M D_{\text {hip-neck }}$ during the study period in. Thin lines age at initiation of HRT (LD group vs HD group: $14.8 \pm 1.4$ vs $14.5 \pm 1.9$ years, $P=0.675)$, initial dose of estrogen $(0.25 \pm 0.12$ vs $0.24 \pm 0.15 \mathrm{mg}, P=0.875)$ and number of years of estrogen treatment before entering the study $(4.4 \pm 1.5$ vs $4.7 \pm 1.4$ years, $P=0.675)$.

\section{Bone mineral density}

At baseline, $\mathrm{BMD}_{\text {hip }}$ and $\mathrm{BMD}_{\text {arm/radius1/3 }}$ were significantly lower in the TS patients combined compared to the controls $\left(\mathrm{BMD}_{\text {hip }}:\right.$ TS vs C: $0.88 \pm 0.14 \mathrm{~g} / \mathrm{cm}^{3}$ vs $0.95 \pm 0.10 \mathrm{~g} / \mathrm{cm}^{3}$, $P=0.037 ; \mathrm{BMD}_{\text {arm } / \mathrm{radius} 1 / 3}$ : TS vs C: $0.62 \pm 0.05 \mathrm{~g} / \mathrm{cm}^{3}$ vs $\left.0.66 \pm 0.05 \mathrm{~g} / \mathrm{cm}^{3}, P=0.009\right)$, whereas all remaining measures of BMD (total, spine and arm/radius UD) were similar among TS and controls (data not shown). At the final visit, there was no significant difference in BMD, total or regional in TS patients compared to controls. However, the TS patients were of course significantly older than the controls at this point (data not shown). The LD and HD TS groups were similar on all measures at baseline (data not shown).

On examining the longitudinal changes in bone mineral density, we found a significant quadratic time effect $(P<0.001)$ in the models for the outcomes $\mathrm{BMD}_{\text {total }}$, $\mathrm{BMD}_{\text {spine, }} \mathrm{BMD}_{\text {hip }}, \mathrm{BMD}_{\text {arm/radiusUD }}, \mathrm{BMD}_{\text {arm/radius } 1 / 3}$ and $\mathrm{VBMD}_{\text {spine }}$ in both treatment groups indicating an increase with a tendency to an attenuated increase toward the end of the study (Fig. 1A and C, Supplementary Figs 1 and 2 ). For the outcome of $\mathrm{vBMD}_{\text {hip }}$, there was no significant quadratic effect $(P=0.4828)$ and hence the increase appeared linear over time (Fig. 1B).

For all seven outcomes, there was no significant effect of estrogen treatment on the yearly BMD measurement (Table 1). Hence, there was no significant difference between the HD group and the LD group in terms of whole body or regional BMD before or after correction for the reduced bone size.

We used multiple linear regression analysis to study the influence of additional parameters on BMD, including BSA, BMI, duration of estrogen treatment before randomization, the presence of spontaneous puberty and BMD at

are the observed data for each patient, straight line (LD group), dotted line (HD group). Thick lines are estimated population means from the mixed model based on all data. (c) Developmental changes BMD arm/radiusuD during the study period in. Thin lines are the observed data for each patient, straight line (LD group), dotted line (HD group). Thick lines are estimated population means from the mixed model based on all data. 
Table 1 Interaction between bone mineral densities and estrogen treatment in both treatment groups. Interaction between bone mineral densities and estrogen treatment at the sites of whole body $\left(B M D_{\text {total }}\right)$, the spine $\left(B M D_{\text {spine }}\right)$, the hip $\left(B M D_{\text {hip }}\right)$, the proximal part (radius $1 / 3)$ of the distal third of the radius $\left(B M D_{\text {arm/radius } 1 / 3}\right)$, the ultradistal part (radius UD) of the distal third of the radius $\left(B M D_{\text {arm/radiusUD }}\right)$, volumetric bone mineral density at the spine $\left(v B M D_{\text {spine }}\right)$, and at the femoral neck $\left(v B M D_{\text {hip neck }}\right)$. Estimated (population-level) effects for the reduced model with no treatment effect with $95 \%$ bootstrapped confidence intervals and the P-value for the null-hypothesis of no treatment effect are shown.

\begin{tabular}{|c|c|}
\hline Outcome & Intercept \\
\hline $\mathrm{BMD}_{\text {total }}$ & $1.04(1.00 ; 1.08)$ \\
\hline BMD $_{\text {spine }}$ & $1.02(0.94 ; 1.08)$ \\
\hline BMD $_{\text {hip }}$ & $0.88(0.82 ; 0.94)$ \\
\hline BMD $_{\text {arm/radiusUD }}$ & $0.43(0.40 ; 0.46)$ \\
\hline $\mathrm{BMD}_{\text {arm} / \text { radius } 1 / 3}$ & $0.63(0.60 ; 0.65)$ \\
\hline vBMD $_{\text {spine }}$ & $0.32(0.30 ; 0.34)$ \\
\hline vBMD $_{\text {hip neck }}$ & $0.32(0.30 ; 0.34)$ \\
\hline
\end{tabular}

\begin{tabular}{c}
\hline Time $\left(* 10^{-2}\right)$ \\
\hline $2.33(1.85 ; 2.84)$ \\
$3.05(2.36 ; 3.77)$ \\
$2.69(2.08 ; 3.33)$ \\
$1.71(1.26 ; 2.15)$ \\
$1.59(1.23 ; 1.94)$ \\
$0.88(0.64 ; 1.13)$ \\
$0.55(0.10 ; 1.04)$ \\
\hline
\end{tabular}

\begin{tabular}{|c|c|}
\hline Time $^{2}\left(* 10^{-2}\right)$ & P-Value \\
\hline$-0.20(-0.28 ;-0.12)$ & 0.2465 \\
\hline$-0.35(-0.46 ;-0.25)$ & 0.5254 \\
\hline$-0.34(-0.44 ;-0.24)$ & 0.3948 \\
\hline$-0.18(-0.25 ;-0.10)$ & 0.761 \\
\hline$-0.14(-0.20 ;-0.07)$ & 0.6644 \\
\hline$-0.11(-0.15 ;-0.06)$ & 0.8807 \\
\hline$-0.03(-0.13 ; 0.06)$ & 0.8877 \\
\hline
\end{tabular}

randomization (at the start of the study). Consistently, at all studied sites, the development in BMD was highly dependent on BMD at randomization, which turned out to be the most influential parameter - thus, the higher BMD at a specific site at inclusion, the higher BMD at that site at the end of the study. Furthermore, in models of $\mathrm{BMD}_{\text {total }}, \mathrm{BMD}_{\text {spine, }}$ $\mathrm{BMD}_{\text {hip }}, \mathrm{BMD}_{\text {arm/radiusUD }}$ and $\mathrm{vBMD}_{\text {spine, }}, \mathrm{BSA}$ and BMI were also positive contributory variables. Additionally, the presence of spontaneous puberty and duration of estrogen treatment before randomization affected $\mathrm{BMD}_{\text {arm/radiusuD, }}$ whereas $\mathrm{BMD}_{\text {arm/radius } 1 / 3}$ was also dependent on $\mathrm{BMI}$ and the presence of spontaneous puberty (Supplementary Table 3). The participants with the karyotype 45,X/46XX $(n=2)$ and spontaneous puberty were not analyzed separately to

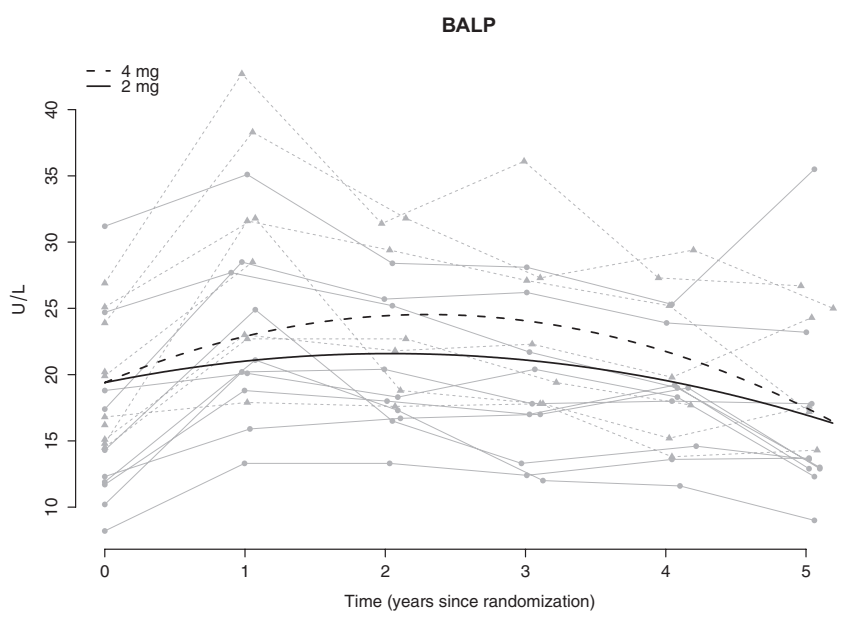

Figure 2

Developmental changes in bone specific alkaline phosphatase (BALP) during the study period. Thin lines are the observed data for each patient, straight line (LD group), dotted line (HD group). Thick lines are estimated population means from the mixed model based on all data. identify a potential different response to estrogen treatment compared to the participants with induced puberty.

\section{Bone markers and hormones}

In both treatment groups, CTX and PINP decreased significantly over time. BALP increased during the first 2 years, and after a plateau, it decreased like the other markers. There were no significant differences in the markers between the treatment groups except for BALP, where the HD group had a significantly higher level throughout the study period compared to the LD group (Fig. 2). In multiple linear regression analyses, we studied possible interacting variables like BSA, BMI, duration of estrogen treatment before randomization, the presence of spontaneous puberty and level of the analyte at randomization. Consistently, we found that the most important variable was the level of the analyte at randomization. However, in addition, for CTX, PINP and BALP, duration of estrogen treatment before randomization and the presence of spontaneous puberty were also contributory variables (Supplementary Table 4).

The level of FSH, LH, estrone sulfate, sex hormonebinding globulin (SHBG), 17OH-progesterone, androstenedione and dihydrotestosterone (DHT) were no different in the two treatment groups (data not shown). The levels of estradiol and estrone were significantly higher in the HD group compared to the LD group $(P=0.0015$ and $P=0.0449$ respectively) after the initial 2 years of treatment (Fig. 3A and B).

\section{Anthropometrics}

TS patients were shorter with comparable body weight, higher BMI and lower BSA when compared to controls 
A

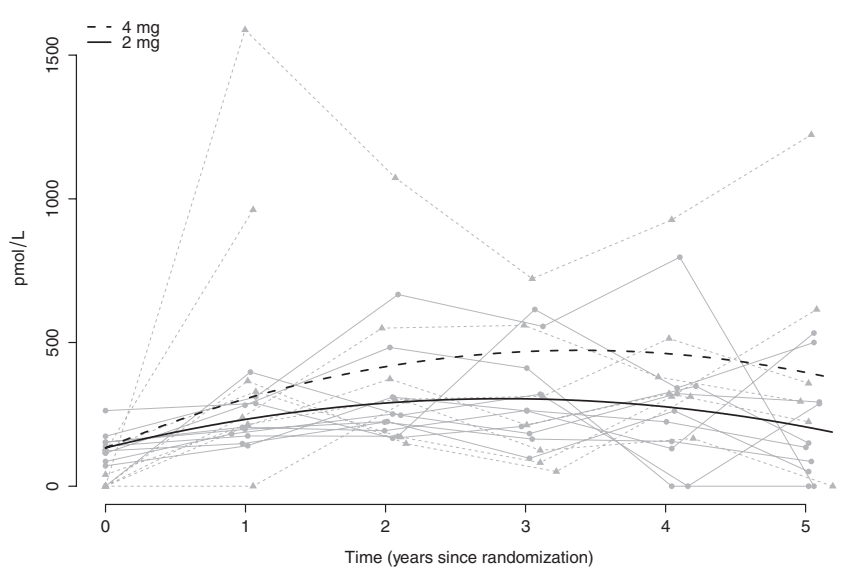

B

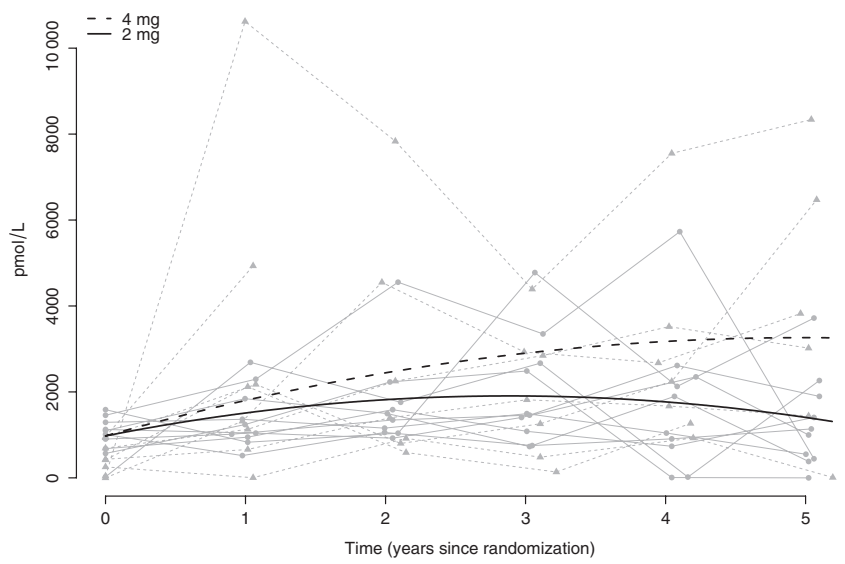

Figure 3

(A) Developmental changes in estradiol during the study period. Thin lines are the observed data for each patient, straight line (LD group), dotted line (HD group). Thick lines are estimated population means from the mixed model based on all data. (B) Developmental changes in estrone during the study period. Thin lines are the observed data for each patient, straight line (LD group), dotted line (HD group). Thick lines are estimated population means from the mixed model based on all data.

(Supplementary Table 2). LBM was lower in TS, whereas FM was similar, resulting in a higher body fat percentage. At baseline, there was no difference in LBM between the LD and HD groups, but at the subsequent yearly visits, there was a significant effect of the interaction between time and treatment $(P=0.0290)$ (Fig. 4). This implies that although LBM remained unchanged throughout the study period in the LD group, it increased in the HD group with an average of $336 \mathrm{~g}$ per year. When studying the longitudinal changes, we found FM and body fat

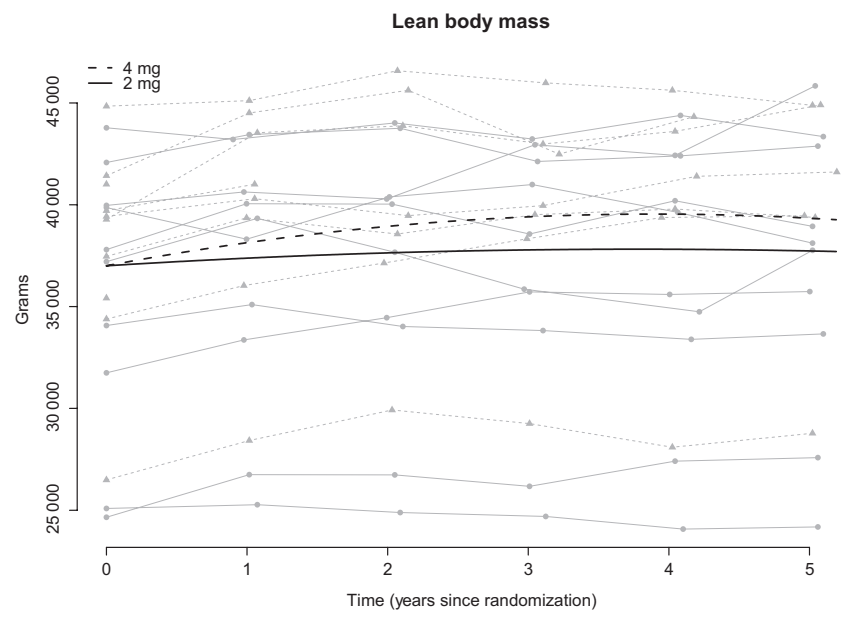

Figure 4

Developmental changes in lean body mass (LBM) during the study period. Thin lines are the observed data for each patient, straight line (LD group), dotted line (HD group). Thick lines are estimated population means from the mixed model based on all data.

percentage to be constant over time with no treatment effect between the treatment groups (Fig. 5A and B).

\section{Discussion}

The principal findings of our study are the selective positive effect of the higher estrogen dose on bone formation and on LBM. However, the former did not translate into a higher BMD indicating that a treatment regimen in TS using the lower estrogen dose is sufficient in terms of accumulating bone mass in late adolescence and early adult years. The present study is to our knowledge the only prospective clinical trial published in which the efficacy of estrogen replacement on bone mineral accrual and body composition in TS is being evaluated in a strict randomized and controlled study design including two comparable groups of TS patients with the only differing factor being the estrogen dose.

The pattern of developmental changes in the BMD over time with an initial increment and eventually stabilization at a consistent level in this group of young women with TS was expected and in accordance with the pattern observed in the general population of healthy young women $(20,21)$. In particular, the increase in the predominately cortical bone sites, hip and lower third radius, is noteworthy as the finding is somewhat contradictory of the general assumption of these areas having a reduced BMD by default in TS due to genetic 
A

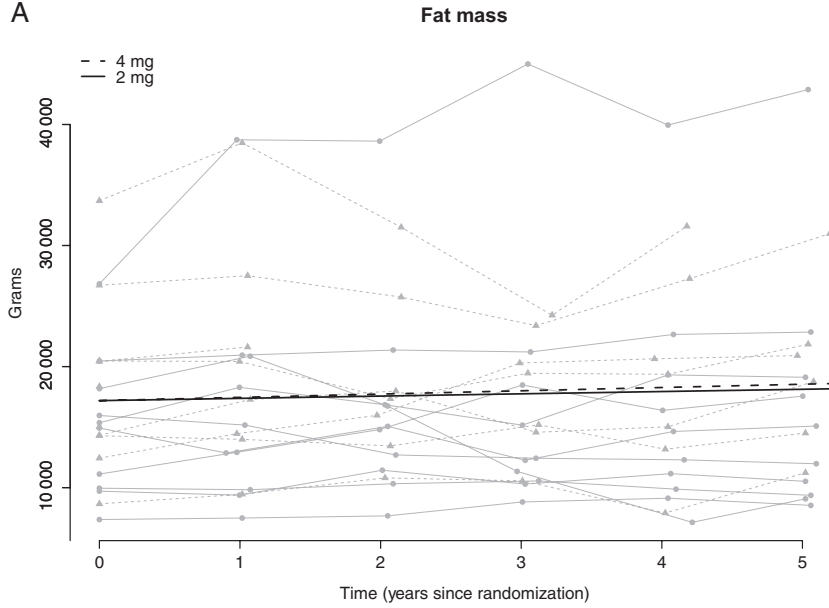

B
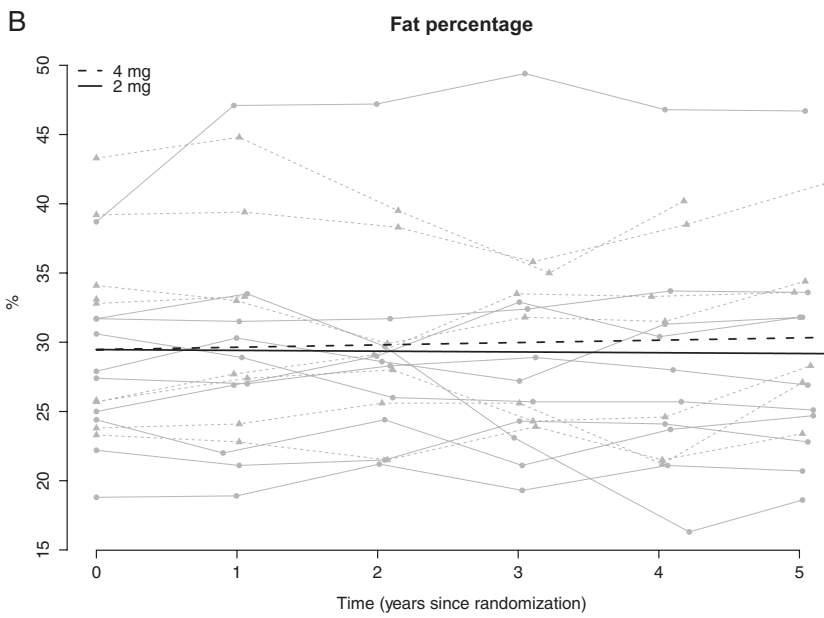

\section{Figure 5}

(A) Developmental changes in fat mass (FM) during the study period. Thin lines are the observed data for each patient, straight line (LD group), dotted line (HD group). Thick lines are estimated population means from the mixed model based on all data. (B) Developmental changes in body fat percentage (Fatt \%) during the study period. Thin lines are the observed data for each patient, straight line (LD group), dotted line (HD group). Thick lines are estimated population means from the mixed model based on all data.

reasons without the potential for improvement neither over time nor under the influence of estrogen $(1,4)$.

Estrogens have well-known strong effects upon trabecular bone. A decreased trabecular volumetric BMD during and after puberty could therefore be expected in hypogonadal TS girls (5) and some studies have indeed shown normal size-adjusted spine BMD before puberty but a lack of adequate pubertal bone acquisition $(22,23$, 24). In contrast, other studies including post-pubertal TS adolescents have found normal trabecular volumetric
BMD at the radius using pQCT $(1,10)$, which can be due to inter-study differences concerning the type, duration and/or dosage of estrogen used, different modalities of and ages at densitometry (5). Here, we show normal average size-adjusted BMD at the spine and the areal BMD at the ultra-distal radius, both sites predominately containing trabecular bone, in comparison with healthy controls on entering the study indicating a sufficient serum estrogen level during pubertal induction in our population of TS adolescent and young women. At the end of the study, we did not see a significant positive effect of the higher estrogen dose on BMD in trabecular bone. In contrast, the most consistent and strongest determinant of BMD at all studied sites was BMD at entry into the study, explaining most of the variance within the TS group, pointing toward the importance of optimizing all components of bone health, including age-appropriate pubertal induction and appropriate $\mathrm{GH}$ treatment inducing a resultant increase in IGF-I, already in childhood and early puberty (25).

The effect of estrogen on the growing skeleton has been extensively reviewed; however, the results have been conflicting (1). It has been assumed that predominantly cortical bone sites in TS are genetically programmed to have reduced BMD without the potential for improvement over time or under the influence of estrogen $(1,17,26$, $27,28,29)$. Our finding of increases in the predominantly cortical bone sites, hip and lower third radius challenge this assumption and are in line with a new study using a true 3D methodology, high-resolution pQCT (hr-pQCT), that found no cortical deficit at least in adult TS (8). At the same time, we found BMD at predominately trabecular bone sites to be comparable to healthy controls after pubertal induction and during the early adult years also irrespectively of the estrogen dose applied.

TS has been characterized as a syndrome of disproportionate anthropometry and body composition, with higher FM and BMI and inappropriately low LBM (10), as also described here. A recent study comparing the transdermal and oral route of administration of 17B-estradiol in a group of TS adolescents found a significant but similar increase in lean body mass by both routes, whereas fat mass remains stable $(30,31)$. We found the same pattern of changes in LBM and FM with an additional positive effect of a higher estrogen dose on LBM. The additional increase in LBM in the HD group during adulthood could play a potential future role as a factor of preventing fracture in older age in TS by improving muscle strength and balance and thereby reducing fall accidents. Increased LBM, and thus probably muscle strength, is also likely to have additional positive 
roles in relation to the frequent occurrence of type 2 diabetes, hypertension, impaired postural balance (32) and obesity (33).

A few studies have looked at markers of bone metabolism to account for differences in the bone phenotype in TS $(18,19)$ and found markers of bone formation and levels of vitamin D to be similar in girls with TS compared to healthy girls. However, markers of bone resorption were elevated in TS. An impaired coupling of bone formation and resorption in girls with TS seems to exist, but it is difficult to discern whether this is related to relative gonadal steroid deficiency, low vitamin D levels, low IGF-1 or a primary gene defect (17). We found both the marker of bone resorption CTX and the marker of bone formation PINP to decrease significantly over time in TS independently of the estrogen dose. At the same time, BALP, marker of bone formation, showed the same pattern of decrease but only after an initial increase during the first 2 years of treatment and with a general significant higher level in the HD group indicating a positive effect of a higher estrogen dose. The question is whether the reduced bone resorption in combination with an estrogen dose-dependent increased bone formation in the same manner predicts a reduced fracture risk independent of BMD in the HD-treated TS and possibly also a higher sitespecific BMD in the future.

In general, the study treatment was well accepted in both treatment groups with good compliance and no reports of major side effects. Even then, four patients, all in the high-dose group, did not complete the entire 5-year treatment plan. The drop-outs, except for one person, were not due to side effects. The unequal distribution of dropout could potentially influence the inter-treatment group analyses as well as the intra-treatment group analyses. However, our statistical model allowed us to utilize patient-specific data until dropout. Theoretical advantages of transdermal (TD) over oral (PO) route of administration include a more physiologic mode of delivery, decreased first pass effect and less variability in markers of hepatic metabolism and IGF1 levels. Whether differences in the mode of estrogen replacement will ultimately translate into meaningful clinical differences in parameters influenced by estrogen in girls and adolescents with TS remains to be proven $(31,34)$.

The strength of the current study is long-term follow-up, which allowed us to precisely map the changes in BMD, body composition and serum markers. It is, however, a drawback that we have not been able follow the control group with yearly measurements.

\section{Conclusion}

The developmental changes in BMD in young adult women with TS are comparable to the pattern observed in the general population of healthy young women, and in the setting of an optimal bone mass accrual during pubertal induction, the benefit of a higher estrogen dose in adolescence and young adulthood seems to be insignificant. Importantly, we show time-dependent physiological increases in both cortical and trabecular bone in TS. A significant and probably clinically relevant increase in LBM was observed with HD estradiol. A future follow-up study elucidating this matter would be relevant as the combined effect of high-dose estrogen on bone and LBM could well have a significant positive impact on the overall morbidity and thereby QoL in TS women at old age.

\section{Supplementary data}

This is linked to the online version of the paper at http://dx.doi.org/10.1530/ EJE-16-0582.

Declaration of interest

C H G reports grants from Novo Nordisk Foundation during the conduct of the study, and C H G has received honorarium from Pfizer in relation to one talk in 2015 .

\section{Funding}

The study was supported by Kaptajnløjtnant Harald Jensen and Wife's foundation, The Research Foundation of the former Frederiksborg County, Queen Louise's Children Hospital Research Foundation, Olga Bryde Nielsen Foundation, Ivan Nielsen's Foundation, The Region 3 Foundation, The Research foundation at Hillerød Hospital, The Novo Nordisk Foundation (personal grant to $\mathrm{C} \mathrm{H} \mathrm{G),} \mathrm{The} \mathrm{East-Danish} \mathrm{Health} \mathrm{Scientific} \mathrm{Research}$ Forum, Torben Iversens Traveling Foundation, Tvergaards Foundation, TODE Traveling Foundation, The Hospital administration and an unrestricted research grant from Novo Nordisk, who generously supplied the study medicine.

\section{Acknowledgements}

Kamma Velin, Merete Pedersen and Gitte Salskov are thanked for expert help, as is The Department of Paediatrics, The Department of Gynecology and Obstetrics, The Department of Quality, Research and Development and the Department of Radiology, Hillerød Hospital. Ole Andersen, is thanked for expert help and support.

\section{References}

1 Holroyd CR, Davies JH, Taylor P, Jameson K, Rivett C, Cooper C \& Dennison EM. Reduced cortical bone density with normal trabecular bone density in girls with Turner syndrome. Osteoporosis International 201021 2093-2099. (doi:10.1007/s00198-010-1170-0) 
2 Gravholt CH, Juul S, Naeraa RW \& Hansen J. Morbidity in Turner syndrome. Journal of Clinical Epidemiology 199851 147-158. (doi:10.1016/S0895-4356(97)00237-0)

3 Gravholt CH, Vestergaard P, Hermann AP, Mosekilde L, Brixen K $\&$ Christiansen JS. Increased fracture rates in Turner's syndrome: a nationwide questionnaire survey. Clinical Endocrinology 200359 89-96. (doi:10.1046/j.1365-2265.2003.01807.x)

4 Pitukcheewanont P, Numbenjapon N, Safani D, Rossmiller S, Gilsanz V \& Costin G. Bone size and density measurements in prepubertal children with Turner syndrome prior to growth hormone therapy. Osteoporosis International 201122 1709-1715. (doi:10.1007/ s00198-010-1375-2)

5 Soucek O, Lebl J, Snajderova M, Kolouskova S, Rocek M, Hlavka Z, Cinek O, Rittweger J \& Sumnik Z. Bone geometry and volumetric bone mineral density in girls with Turner syndrome of different pubertal stages. Clinical Endocrinology 201174 445-452. (doi:10.1111/ j.1365-2265.2010.03955.x)

6 Bakalov VK, Chen ML, Baron J, Hanton LB, Reynolds JC, Stratakis CA, Axelrod LE \& Bondy CA. Bone mineral density and fractures in Turner syndrome. American Journal of Medicine 2003115 259-264. (doi:10.1016/S0002-9343(03)00364-4)

7 Andrade AC, Baron J, Manolagas SC, Shaw NJ, Rappold GA, Donaldson MD, Gault EJ \& Sävendahl L Hormones and genes of importance in bone physiology and their influence on bone mineralization and growth in Turner syndrome. Hormone Research in Paediatrics 201073 161-165. (doi:10.1159/000284356)

8 Hansen S, Brixen K \& Gravholt CH. Compromised trabecular microarchitecture and lower finite element estimates of radius and tibia bone strength in adults with turner syndrome: a cross-sectional study using high-resolution-pQCT. Journal of Bone and Mineral Research 201227 1794-1803. (doi:10.1002/jbmr.1624)

9 Gravholt CH, Naeraa RW, Fisker S \& Christiansen JS. Body composition and physical fitness are major determinants of the growth hormone-insulin-like growth factor axis aberrations in adult Turner's syndrome, with important modulations by treatment with 17 beta-estradiol. Journal of Clinical Endocrinology and Metabolism 1997 82 2570-2577. (doi:10.1210/jc.82.8.2570)

10 Gravholt CH, Hjerrild BE, Mosekilde L, Hansen TK, Rasmussen LM, Frystyk J, Flyvbjerg A \& Christiansen JS. Body composition is distinctly altered in Turner syndrome: relations to glucose metabolism, circulating adipokines, and endothelial adhesion molecules. European Journal of Endocrinology 2006155 583-592. (doi:10.1530/eje.1.02267)

11 Bechtold S, Rauch F, Noelle V, Donhauser S, Neu CM, Schoenau E $\&$ Schwarz HP. Musculoskeletal analyses of the forearm in young women with Turner syndrome: a study using peripheral quantitative computed tomography. Journal of Clinical Endocrinology and Metabolism 200186 5819-5823. (doi:10.1210/jcem.86.12.8063)

12 Hogler W, Briody J, Moore B, Garnett S, Lu PW \& Cowell CT. Importance of estrogen on bone health in Turner syndrome: a cross-sectional and longitudinal study using dual-energy X-ray absorptiometry. Journal of Clinical Endocrinology and Metabolism 2004 89 193-199. (doi:10.1210/jc.2003-030799)

13 Soucek O, Matyskova J, Anliker E, Toigo M, Hlavka Z, Lebl J \& Sumnik Z. The muscle-bone interaction in Turner syndrome. Bone 201574 160-165. (doi:10.1016/j.bone.2015.01.017)

14 Cleemann L, Hjerrild BE, Lauridsen AL, Heickendorff L, Christiansen JS, Mosekilde L \& Gravholt CH. Long-term hormone replacement therapy preserves bone mineral density in Turner syndrome. European Journal of Endocrinology 2009161 251-257. (doi:10.1530/EJE-09-0020)

15 Khastgir G, Studd JW, Fox SW, Jones J, Alaghband-Zadeh J \& Chow JW. A longitudinal study of the effect of subcutaneous estrogen replacement on bone in young women with Turner's syndrome. Journal of Bone and Mineral Research 200318 925-932. (doi:10.1359/ jbmr.2003.18.5.925)
16 Gravholt $\mathrm{CH}$, Riis AL, Moller N \& Christiansen JS. Protein metabolism in Turner syndrome and the impact of hormone replacement therapy. Clinical Endocrinology 200767 413-418. (doi:10.1111/j.13652265.2007.02902.x)

17 Marino R \& Misra M. Bone health in primary ovarian insufficiency. Seminars in Reproductive Medicine 201129 317-327. (doi:10.105 5/s-0031-1280917)

18 Gravholt CH, Lauridsen AL, Brixen K, Mosekilde L, Heickendorff L \& Christiansen JS. Marked disproportionality in bone size and mineral, and distinct abnormalities in bone markers and calcitropic hormones in adult turner syndrome: a cross-sectional study. Journal of Clinical Endocrinology and Metabolism 200287 2798-2808. (doi:10.1210/ jcem.87.6.8598)

19 Cleemann L, Holm K, Kobbernagel H, Skouby SO, Kristensen B, Smedegaard H, Andersson AM, Cohen A \& Gravholt CH. Normal tempo of bone formation in Turner syndrome despite signs of accelerated bone resorption. Hormone Research in Paediatrics 201176 193-201. (doi:10.1159/000329046)

20 Schoenau E, Saggese G, Peter F, Baroncelli GI, Shaw NJ, Crabtree NJ, Zadik K, Neu CM, Noordam C, Radetti G et al. From bone biology to bone analysis. Hormone Research 200461 257-269. (doi:10.1159/000076635)

21 Schoenau E, Land C, Stabrey A, Remer T \& Kroke A. The bone mass concept: problems in short stature. European Journal of Endocrinology 2004151 (Supplement 1) S87-S91. (doi:10.1530/eje.0.151S087)

22 Ross JL, Long LM, Feuillan P, Cassorla F \& Cutler GB Jr. Normal bone density of the wrist and spine and increased wrist fractures in girls with Turner's syndrome. Journal of Clinical Endocrinology and Metabolism 199173 355-359. (doi:10.1210/jcem-73-2-355)

23 Shaw NJ, Rehan VK, Husain S, Marshall T \& Smith CS. Bone mineral density in Turner's syndrome - a longitudinal study. Clinical Endocrinology 199747 367-370. (doi:10.1046/j.13652265.1997.2791084.x)

24 Nanao K, Tsuchiya Y, Kotoh S \& Hasegawa Y. Low vertebral cancellous bone density in peripubertal girls with Turner's syndrome and boys with hypogonadism. Journal of Pediatric Endocrinology and Metabolism 200215 1537-1542. (doi:10.1515/ipem.2002.15.9.1537)

25 Bonjour JP \& Chevalley T. Pubertal timing, bone acquisition, and risk of fracture throughout life. Endocrine Reviews 201435 820-847. (doi:10.1210/er.2014-1007)

26 Wang Q, Alen M, Nicholson PH, Halleen JM, Alatalo SL, Ohlsson C, Suominen H \& Cheng S. Differential effects of sex hormones on periand endocortical bone surfaces in pubertal girls. Journal of Clinical Endocrinology and Metabolism 200691 277-282. (doi:10.1210/jc.20051608)

27 Bakalov VK, Axelrod L, Baron J, Hanton L, Nelson LM, Reynolds JC, Stratakis CA, Axelrod LE \& Bondy CA. Selective reduction in cortical bone mineral density in turner syndrome independent of ovarian hormone deficiency. Journal of Clinical Endocrinology and Metabolism 200388 5717-5722. (doi:10.1210/jc.2003-030913)

28 Nadeem M \& Roche EF. Bone health in children and adolescent with Turner syndrome. Journal of Pediatric Endocrinology and Metabolism 201225 823-833. (doi:10.1515/jpem-2012-0088)

29 Vanderschueren D, Venken K, Ophoff J, Bouillon R \& Boonen S. Clinical review: sex steroids and the periosteum - reconsidering the roles of androgens and estrogens in periosteal expansion. Journal of Clinical Endocrinology and Metabolism 200691 378-382. (doi:10.1210/ jc.2005-1766)

30 Isotton AL, Wender MC, Casagrande A, Rollin G \& Czepielewski MA. Effects of oral and transdermal estrogen on IGF1, IGFBP3, IGFBP1, serum lipids, and glucose in patients with hypopituitarism during GH treatment: a randomized study. European Journal of Endocrinology 2012 166 207-213. (doi:10.1530/EJE-11-0560)

31 Torres-Santiago L, Mericq V, Taboada M, Unanue N, Klein KO, Singh R, Hossain J, Santen RJ, Ross JL \& Mauras N. Metabolic effects of oral versus transdermal 17beta-estradiol ( $\mathrm{E}(2))$ : a randomized clinical 
trial in girls with Turner syndrome. Journal of Clinical Endocrinology and Metabolism 201398 2716-2724. (doi:10.1210/jc.2012-4243)

32 Wahlberg J, Sydsjo G, Ledin T, Bagesund M \& Ekman B. Impaired postural balance in turner syndrome. Hormone and Metabolic Research 201345 537-540. (doi:10.1055/s-0033-1333718)

33 Mortensen KH, Andersen NH \& Gravholt CH. Cardiovascular phenotype in Turner syndrome - integrating cardiology, genetics, and endocrinology. Endocrine Reviews 201233 677-714. (doi:10.1210/ er.2011-1059)

34 Taboada M, Santen R, Lima J, Hossain J, Singh R, Klein KO \& Mauras N. Pharmacokinetics and pharmacodynamics of oral and transdermal 17\{beta\} estradiol in girls with turner syndrome. Journal of Clinical Endocrinology and Metabolism 201196 3502-3510. (doi:10.1210/jc.2011-1449)

Received 10 July 2016

Revised version received 11 November 2016

Accepted 23 November 2016 